\title{
Remoción de Carbono y Nitratos de Vertidos de la Industria Salmonera usando Reactores Tubulares
}

\author{
César E. Huiliñir, Estrella R. Aspé y Marlene D. Roeckel* \\ Universidad de Concepción, Facultad de Ingeniería, Departamento de Ingeniería Química, \\ Casilla 160-C, Concepción-Chile (e-mail: mroeckel@udec.cl)
}

*autor a quien debe ser dirigida la correspondencia

\begin{abstract}
Resumen
Se estudió la remoción simultánea de carbono y nitratos de vertidos salmoneros salinos ( $24 \mathrm{~g} / \mathrm{L})$ en reactores tubulares con biomasa adherida a $37^{\circ} \mathrm{C}$. Se trabajó a velocidades de carga orgánica de $1.49 \mathrm{~kg} \mathrm{DQO} / \mathrm{m}^{3} \mathrm{~d}$ (condición 1) y $11.46 \mathrm{~kg} \mathrm{DQO} / \mathrm{m}^{3} \mathrm{~d}$ (condición 2) en un rango entre 2800 a 5000 $\mathrm{mg} \mathrm{DQO} / \mathrm{L}$, con relaciones $\mathrm{C} / \mathrm{N}$ de $18,39,80$ y $142 \mathrm{mg} \mathrm{DQO} / \mathrm{mg} \mathrm{N}-\mathrm{NO}_{3}{ }^{-}$. Para ambas condiciones, se logró $98 \%$ de reducción de nitratos, mientras que la remoción de materia orgánica fue de hasta $88 \%$ para la condición 1 , y menor al 50 \% para la condición 2 . El amonio se produjo mayormente por la digestión anaeróbica y no por la reacción de nitrato a amonio. Se concluye que es factible remover nitratos de residuos industriales salmoneros, observando que a menor velocidad de carga orgánica se logra mayor eficiencia en la remoción de materia orgánica.
\end{abstract}

Palabras clave: desnitrificación, digestión anaerobia, reactor tubular, industria salmonera, biomasa adherida

\section{Removal of Nitrate and Organic Matter from Salmon Industry Wastewaters using Plug Flow Reactors}

\begin{abstract}
The simultaneous removal of carbon and nitrogen from saline salmon plant effluents in fixed biofilm tubular reactors were studied. Two organic load rates were used: $1.49 \mathrm{~kg} \mathrm{COD} / \mathrm{m}^{3} \mathrm{~d}$ (situation 1) and $11.46 \mathrm{~kg} \mathrm{COD} / \mathrm{m}^{3} \mathrm{~d}$ (situation 2) for 2800 and $5000 \mathrm{mg} \mathrm{COD} / \mathrm{L}$ respectively, using $\mathrm{C} / \mathrm{N}$ ratios of $18,39,80$ and $142 \mathrm{mg} \mathrm{COD} / \mathrm{mg} \mathrm{N}^{-N^{-}}{ }_{3}^{-}$. The nitrate abatement was $98 \%$ in both cases while up to $88 \%$ removal for condition 1 and less than $50 \%$ removal for condition 2 were achieved. Ammonia was mainly produced by the anaerobic digestion and not by nitrate reduction to ammonia. It is concluded that it is technically possible to remove nitrates form saline salmon plant effluents observing that as the organic load rates decreases the removal efficiency increases.
\end{abstract}

Keywords: denitrification, anaerobic digestion, plug flow reactor, salmon industry, biofilm 


\section{INTRODUCCIÓN}

La presencia de altas concentraciones de carbono y nitrógeno orgánico caracterizan el residuo industrial líquido (RIL) producido en la industria procesadora de recursos marinos. Una de las alternativas de tratamiento de este tipo de residuos es el proceso en dos etapas: desnitrificación anóxica / digestión anaeróbica y aerobiosis (Im et al., 2001). A diferencia del proceso tradicional, en esta propuesta se integran directamente las etapas desnitrificante y anaeróbica en un solo reactor, como se muestra en la Fig. 1. siendo estas etapas las presentadas en este trabajo.

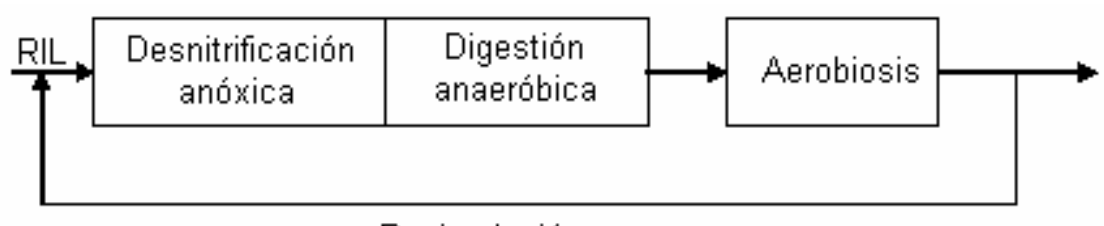

Recirculación

Fig. 1: Proceso alternativo de tratamiento biológico para residuos carbonosos y nitrogenados.

El esquema presentado en la Fig. 1 utiliza en primer lugar la fuente de carbono para la desnitrificación, y el remanente es utilizado para la digestión anaerobia, siendo esto una ventaja en comparación con el primer proceso, debido a que no es necesario agregar una fuente de carbono adicional a la etapa de desnitrificación. Además, al tener una etapa menos, por la eliminación de la etapa separada para la desnitrificación, se disminuyen los costos tanto de instalación como de funcionamiento de estos reactores (Im et al., 2001; Hendriksen y Ahring, 1996; Akunna et al., 1994).

Por otro lado, la utilización de reactores tubulares de lecho relleno con biopelícula resulta muy adecuado para este tipo de proceso, ya que la biomasa adherida crea un micro y macroambiente dentro del reactor que permite el crecimiento de las bacterias en distintas zonas donde se favorece su actividad metabólica (Bernet et al., 2000), permitiendo la regulación natural del límite desnitrificante - D.A. al consumirse primero los nitritos y nitratos y, concomitantemente, sea consumida la materia orgánica como dador de electrones (Mosquera-Corral et al., 2001; Bernet et al., 1996a; Akunna et al., 1992).

Uno de los problemas de este proceso desnitrificante/anaeróbico es la competencia entre la reacción de desnitrificación y la reacción desasimilatoria de nitrato a amonio (RDNA). Varios autores (Bernet et al., 1996b; Akunna et al., 1992) han determinado que el aumento de la RDNA se produce en presencia de materia orgánica fermentable y altas relaciones $\mathrm{C} / \mathrm{N}$, por lo que es de interés determinar si existe este efecto en reactores con biomasa adherida para sustrato proteico. Por otro lado, aún no existe claridad respecto al efecto de otros parámetros como lo es la velocidad de carga orgánica (VCO) y altas relaciones $\mathrm{C} / \mathrm{N}$ sobre la eficiencia del reactor. Chen et al. (1997), trabajando con metanol como fuente de carbono, determinaron que tiempos de residencia hidráulicos (TRH) $>2 \mathrm{~h}$ entregan eficiencias sobre el $90 \%$; sin embargo, su estudio sólo se hizo a bajas relaciones $\mathrm{C} / \mathrm{N}$ y

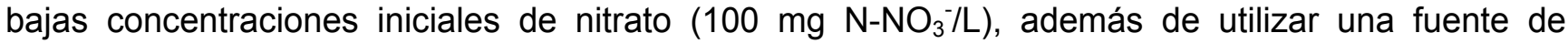
carbono no fermentable. Mosquera-Corral et al. (2001), utilizando efluentes de la industria conservera como fuente de carbono, determinaron que es factible el proceso de desnitrificación para una VCO entre 1-1,25 g DQO/ $\mathrm{L} d$, pero sólo estudiaron el proceso a relaciones $\mathrm{C} / \mathrm{N}$ cercana a la estequiométrica, no analizando relaciones $\mathrm{C} / \mathrm{N}$ superiores. Ruiz et al. (2006), estudiaron el efecto de la relación $\mathrm{C} / \mathrm{N}$ en un reactor UASB, sin embargo, sólo trabajaron a una VCO fija de $7,5 \mathrm{~g} \mathrm{DQO/L} \mathrm{d.}$ De esta forma, es necesario determinar los posibles escenarios en función de la relación C/N y VCO para la eficiente remoción de carbono y nitrógeno de sustratos salinos y proteicos.

El presente trabajo estudió el comportamiento de un reactor tubular de biomasa adherida desnitrificante/anaeróbico a diferentes relaciones $\mathrm{C} / \mathrm{N}$ y velocidades de carga orgánica (VCO), con el fin de obtener un escenario óptimo para la eliminación simultánea de materia orgánica y nitrogenada desde vertidos salmoneros. 


\section{METODOLOGÍA}

Se utilizaron 4 reactores filtro sin recirculación desnitrificantes/anaeróbicos $(\mathrm{V}=6,818 \mathrm{~L} ; \mathrm{H}=1,65 \mathrm{~m}$; diámetro interno $=0,071 \mathrm{~m}$ ) rellenos con PVC rugoso, de área específica, $438 \mathrm{~m}^{2} / \mathrm{m}^{3}$. La porosidad del reactor fue de 0,72 . Los reactores fueron alimentados con una mezcla $(\mathrm{v} / \mathrm{v})$ de $50 \%$ RIL salmonero y $50 \%$ de sustrato sintético. La composición del promedio del efluente industrial fue $(\mathrm{g} / \mathrm{L})$ : $\mathrm{N}-\mathrm{NO}_{3}{ }^{-}, 0 ; \mathrm{N}^{-\mathrm{NO}_{2}}{ }^{-}, 0 ; \mathrm{N}^{-\mathrm{NH}_{4}}{ }^{+}, 0,023$; nitrógeno total Kjeldahl, 10.755; DQO, 6.469; $\mathrm{NaCl}$, 24; proteína, 2.536; $\mathrm{SO}_{4}{ }^{-2}, 0,001$. La composición del sustrato sintético fue $(\mathrm{g} / \mathrm{L}): \mathrm{KH}_{2} \mathrm{PO}_{4}, 3 ; \mathrm{NaHCO}_{3}, 0,4 ;$ $\mathrm{MgSO}_{4} \cdot 7 \mathrm{H}_{2} \mathrm{O}, 0,005 ; \mathrm{NaCl}, 24$; extracto de levadura, 0,05; peptona caseína, 0,12; $\mathrm{FeSO}_{4} \cdot 7 \mathrm{H}_{2} \mathrm{O}$, 0,0011; $\mathrm{CaCl}_{2}, 0,0005$; ácido acético 17,5 M, 1,22 mL/L.

Las condiciones de operación de los reactores se muestran en la Tabla 1, donde el tiempo de residencia hidráulico se calculó como:

$\mathrm{TRH}=\frac{\mathrm{V} \cdot \varepsilon}{\mathrm{Q}}$

donde:

$Q=$ caudal de entrada, $L / d$.

$\varepsilon=$ porosidad del reactor.

$\mathrm{V}=$ volumen del reactor, $\mathrm{L}$.

Tabla 1: Condiciones de funcionamiento de reactores desnitrificantes/anaeróbicos utilizados en este trabajo.

\begin{tabular}{|c|c|c|c|c|}
\hline \multicolumn{5}{|c|}{ Etapa 1} \\
\hline Parámetro & Reactor 1 & Reactor 2 & Reactor 3 & Reactor 4 \\
\hline Relación $\mathrm{C} / \mathrm{N}\left[\mathrm{mg} \mathrm{DQO} / \mathrm{mg} \mathrm{N}^{\left.-\mathrm{NO}_{3}{ }^{-}\right]}\right.$ & 80 & 142 & 39 & 18 \\
\hline Concentración DQO [mg DQO/L] & 2840 & 2840 & 2840 & 2840 \\
\hline Concentración proteína [mg/L] & 2600 & 2600 & 2600 & 2600 \\
\hline Concentración Nitrato [mg N-NO$\left.{ }_{3}^{-} / \mathrm{L}\right]$ & 36 & 20 & 72 & 160 \\
\hline Tiempo residencia hidráulico [d] & 2,6 & 2,6 & 2,6 & 2,6 \\
\hline $\begin{array}{l}\text { Velocidad de carga orgánica (VCO) } \\
{\left[\mathrm{kg} \mathrm{DQO} / \mathrm{m}^{3} \mathrm{~d}\right]}\end{array}$ & 1,1 & 1,1 & 1,1 & 1,1 \\
\hline $\begin{array}{l}\text { Velocidad de carga nitrogenada (VCN) } \\
{\left[\mathrm{kg} \mathrm{N}-\mathrm{NO}_{3}^{-} / \mathrm{m}^{3} \mathrm{~d}\right]}\end{array}$ & 0,014 & 0,008 & 0,03 & 0,062 \\
\hline \multicolumn{5}{|c|}{ Etapa 2} \\
\hline Parámetro & Reactor 1 & Reactor 2 & Reactor 3 & Reactor 4 \\
\hline Relación $\mathrm{C} / \mathrm{N}\left[\mathrm{mg} \mathrm{DQO} / \mathrm{mg} \mathrm{N}^{\left.-\mathrm{NO}_{3}{ }^{-}\right]}\right.$ & 80 & 142 & 39 & 18 \\
\hline Concentración DQO [mg DQO/L] & 5000 & 5000 & 5000 & 5000 \\
\hline Concentración proteína [mg/L] & 4500 & 4500 & 4500 & 4500 \\
\hline 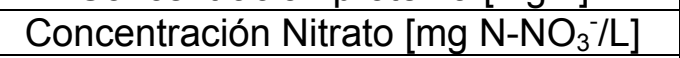 & 65 & 35 & 127 & 284 \\
\hline Tiempo residencia hidráulico [d] & 0,5 & 0,5 & 0,5 & 0,5 \\
\hline $\begin{array}{c}\text { Velocidad de carga orgánica (VCO) } \\
{\left[\mathrm{kg} \mathrm{DQO} / \mathrm{m}^{3} \mathrm{~d}\right]}\end{array}$ & 10 & 10 & 10 & 10 \\
\hline $\begin{array}{l}\text { Velocidad de carga nitrogenada (VCN) } \\
{\left[\mathrm{kg} \mathrm{N}-\mathrm{NO}_{3}-1 \mathrm{~m}^{3} \mathrm{~d}\right]}\end{array}$ & 0,13 & 0,07 & 0,25 & 0,57 \\
\hline
\end{tabular}

Las velocidades de carga orgánica ensayadas fueron escogidas dentro del rango existente en la literatura, los cuales varían entre 1 y $20 \mathrm{~kg} \mathrm{DQO} / \mathrm{m}^{3} \mathrm{~d}$. En este sentido, las concentraciones de DQO (2800 y 5000) ensayadas están dentro del rango de DQO de un efluente pesquero, el cual fluctúa dentro de los 2-6 g DQO/L (Vidal et al., 1997). Las concentraciones de nitrato utilizadas variaron en función de la relación $\mathrm{C} / \mathrm{N}$ utilizada y éstas fueron escogidas de acuerdo a lo estudiado por otros 
autores (Baloch et al. 2006; Bernet et al. 2000; Bernet et al. 1996b); ó considerando la transformación completa del amonio generado en la digestión anaeróbica, que puede alcanzar concentraciones de hasta $1,5 \mathrm{~g}$ de N/L (Aspé et al., 1997).

Los reactores fueron operados por $200 \mathrm{~d}$. Logrado el estado estacionario, se tomaron muestras de la alimentación, de la descarga y a lo largo de los reactores y se filtraron. A las muestras filtradas se les determinó los siguientes parámetros: Carbono Orgánico Total (COT), Demanda Química de Oxígeno (DQO), Nitrógeno Total Kjeldahl (NTK) pH, Nitrato, Nitrito, nitrógeno total amoniacal y alcalinidad. El pH, DQO y NTK fueron medidos por Métodos Estándar (APHA, 1992). El nitrito, nitrato y nitrógeno total amoniacal fueron medidos por un equipo de análisis de inyección de flujo (Foss Tecator, Hoganas, Suecia), de acuerdo a Sánchez et al. (2005a). El nitrógeno total amoniacal es la suma del amonio $\left(\mathrm{N}-\mathrm{NH}_{4}{ }^{+}\right)$y el amoniaco $\left(\mathrm{N}-\mathrm{NH}_{3}\right)$, ambos expresados como nitrógeno. El COT fue medido en un equipo analizador de COT (Shimadzu, modelo TOC-5000 ${ }^{\mathrm{a}}$, Japón). La alcalinidad debida a los AGV (alcalinidad intermedia, Al), la alcalinidad debida al bicarbonato (AP) y la alcalinidad total (AT) fueron determinadas de acuerdo a Ripley et al. (1986). La relación de alcalinidad RA, se definió como la relación entre la alcalinidad debida a los AGV (AI) y la alcalinidad total (AT), Al/AT (Campos, 2001).

La relación entre DQO y COT se determinó experimentalmente y resultó ser:

$$
\text { g DQO/g TOC }=2,185
$$

Las proteínas fueron medidas por el método de Lowry (González et al., 2005). La eficiencia de remoción de carbono y nitrógeno se determinó a través de las siguientes ecuaciones:

Remoción de $\mathrm{DQO}(\%)=\frac{[D Q O]_{A}-[D Q O]_{E}}{[D Q O]_{A}} \cdot 100$
Remoción de $\mathrm{N}-\mathrm{NO}_{3}^{-}(\%)=\frac{\left[N-N O_{3}^{-}\right]_{A}-\left[N-N O_{3}^{-}\right]_{E}}{\left[N-N O_{3}^{-}\right]_{A}} \cdot 100$

donde

$[D Q O]_{A}=$ concentración de $\mathrm{DQO}$ en el influente, $\mathrm{mg} / \mathrm{L}$.

$[D Q O]_{E}=$ concentración de $\mathrm{DQO}$ en el efluente, $\mathrm{mg} / \mathrm{L}$.

$\left[\mathrm{N}-\mathrm{NO}_{3}^{-}\right]_{A}=$ concentración de $\mathrm{N}-\mathrm{NO}_{3}^{-}$en el influente, $\mathrm{mg} / \mathrm{L}$.

$\left[\mathrm{N}-\mathrm{NO}_{3}^{-}\right]_{E}=$ concentración de $\mathrm{N}-\mathrm{NO}_{3}^{-}$en el efluente, $\mathrm{mg} / \mathrm{L}$

La velocidad de remoción de nitrato (VRN) se determinó mediante la siguiente ecuación:

$\operatorname{VRN}=\frac{\left[N-N O_{3}^{-}\right]_{A}-\left[N-N O_{3}^{-}\right]_{E}}{T R H}$

donde

$T R H=$ tiempo de residencia hidráulico, $\mathrm{d}$.

El balance de nitrógeno total amoniacal se determinó suponiendo que la asimilación de nitrógeno es despreciable, de esta forma:

$Q \cdot\left[N-N H_{4}^{+}\right]_{E}=Q \cdot\left[N-N H_{4}^{+}\right]_{A}+Q \cdot\left[N-N H_{4}^{+}\right]_{R D N A}+Q \cdot\left[N-N H_{4}^{+}\right]_{D A}$

donde

$Q=$ caudal de entrada y salida, $L / d$ 
$\left[\mathrm{N}-\mathrm{NH}_{4}^{+}\right]_{E}=$ concentración de nitrógeno total amoniacal a la salida del reactor, $\mathrm{mg} / \mathrm{L}$.

$\left[\mathrm{N}-\mathrm{NH}_{4}^{+}\right]_{A}=$ concentración de nitrógeno total amoniacal a la entrada del reactor, $\mathrm{mg} / \mathrm{L}$.

$\left[\mathrm{N}-\mathrm{NH}_{4}^{+}\right]_{R D N A}=$ concentración de nitrógeno total amoniacal producida por RDNA, mg/L.

$\left[\mathrm{N}-\mathrm{NH}_{4}^{+}\right]_{D A}=$ concentración de nitrógeno total amoniacal producida por acidogénesis, $\mathrm{mg} / \mathrm{L}$.

La concentración de nitrógeno total amoniacal producida por acidogénesis fue calculada considerando que el $16 \%$ de las proteínas corresponde a nitrógeno y que toda la proteína hidrolizada es transformada a ácidos grasos volátiles y amonio:

$\left[N-N H_{4}^{+}\right]_{D A}=0,16 \cdot\left(C_{p r o t, A}-C_{p r o t, E}\right)$

donde $C_{\text {prot,A}}$ y $C_{\text {prot, },}$ son las concentraciones de entrada y salida de proteínas, respectivamente. De esta forma, la masa de nitrógeno total amoniacal producido por RDNA se calcula como:

$Q \cdot\left[N-N H_{4}^{+}\right]_{R D N A}=Q \cdot\left[N-N H_{4}^{+}\right]_{A}+Q \cdot\left[N-N H_{4}^{+}\right]_{D A}-Q \cdot\left[N-N H_{4}^{+}\right]_{E}$

Se consideró significativo el $\mathrm{N}-\mathrm{NH}_{4}{ }^{+}$por RDNA si el porcentaje generado es mayor al $5 \%$, que es el error del método considerado en las mediciones.

\section{RESULTADOS Y DISCUSIÓN}

\section{Comportamiento dinámico de los reactores}

El comportamiento dinámico de los reactores en ambas etapas de experimentación para las variables $\mathrm{DQO}$ y N-NH${ }_{4}^{+}$se muestra en las Fig. 2 y 3 para una relación $\mathrm{C} / \mathrm{N}$ de 80 . Los reactores con relación $\mathrm{C} / \mathrm{N}$ de 18, 39 y 142 presentaron un comportamiento similar. Se aprecia que en la primera etapa, los reactores mostraron una clara disminución del DQO de descarga en el tiempo, correspondiente a una degradación de 80 hasta $88 \%$, estabilizándose aproximadamente a los 40 días de experimentación. No se encontró $\mathrm{N}_{-} \mathrm{NO}_{3}{ }^{-}$ni N-NO${ }_{2}^{-}$en el efluente para esta etapa, por lo que la remoción de estas formas nitrogenadas fue completa. El N- $\mathrm{NH}_{4}{ }^{+}$varió en función de la degradación de materia orgánica, promediando los $350 \mathrm{mg} \mathrm{N}-\mathrm{NH}_{4}{ }^{+} / \mathrm{L}$ para todas las relaciones $\mathrm{C} / \mathrm{N}$ (Fig. 3). El pH de entrada para la primera etapa se mantuvo relativamente constante $(6,5)$, al igual que el $\mathrm{pH}$ de salida, el cual resultó ser más alto, bordeando la neutralidad.

En la segunda etapa, a mayor VCO, no se observó una disminución pronunciada del DQO en el sistema, lográndose el estado estacionario en mucho mayor tiempo que en la etapa 1 (100 días más). La remoción de materia orgánica no superó el $50 \%$ en esta etapa, para todas las relaciones $\mathrm{C} / \mathrm{N}$.

Los valores de $\mathrm{N}^{-N_{3}}{ }_{3}^{-}$y $\mathrm{N}_{-} \mathrm{NO}_{2}{ }^{-}$, al igual que la etapa anterior, fueron muy bajos (< a $4 \mathrm{mg} \mathrm{N}-\mathrm{NO}_{2}{ }^{-} / \mathrm{L}$ ) a la salida del reactor para todas las relaciones $\mathrm{C} / \mathrm{N}$, lográndose una eficiencia superior al $98 \%$. El

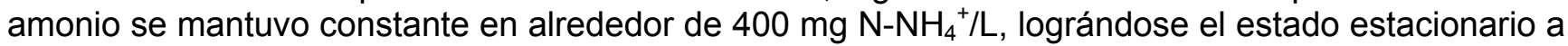
los 40 días de experimentación en la segunda etapa. El pH de salida aumentó con respecto al de entrada $(\mathrm{pH}=6,5)$, siendo mucho más irregular que en la etapa anterior, fluctuando entre 6,8 y 6,4. De esta forma, se puede decir que el incremento en la VCO repercute en un mayor tiempo de estabilización antes del estado estacionario.

\section{Análisis en estado estacionario}

En las Figuras 4,5 y 6 , se presentan los perfiles de concentración de $\mathrm{DQO}, \mathrm{N}_{-} \mathrm{NO}_{3}^{-}$y $\mathrm{pH}$, obtenidos para el reactor con relación $\mathrm{C} / \mathrm{N}$ igual a 80 . Los perfiles obtenidos para los reactores con relación $\mathrm{C} / \mathrm{N}$ de 18,39 y 142 presentan un comportamiento similar. 


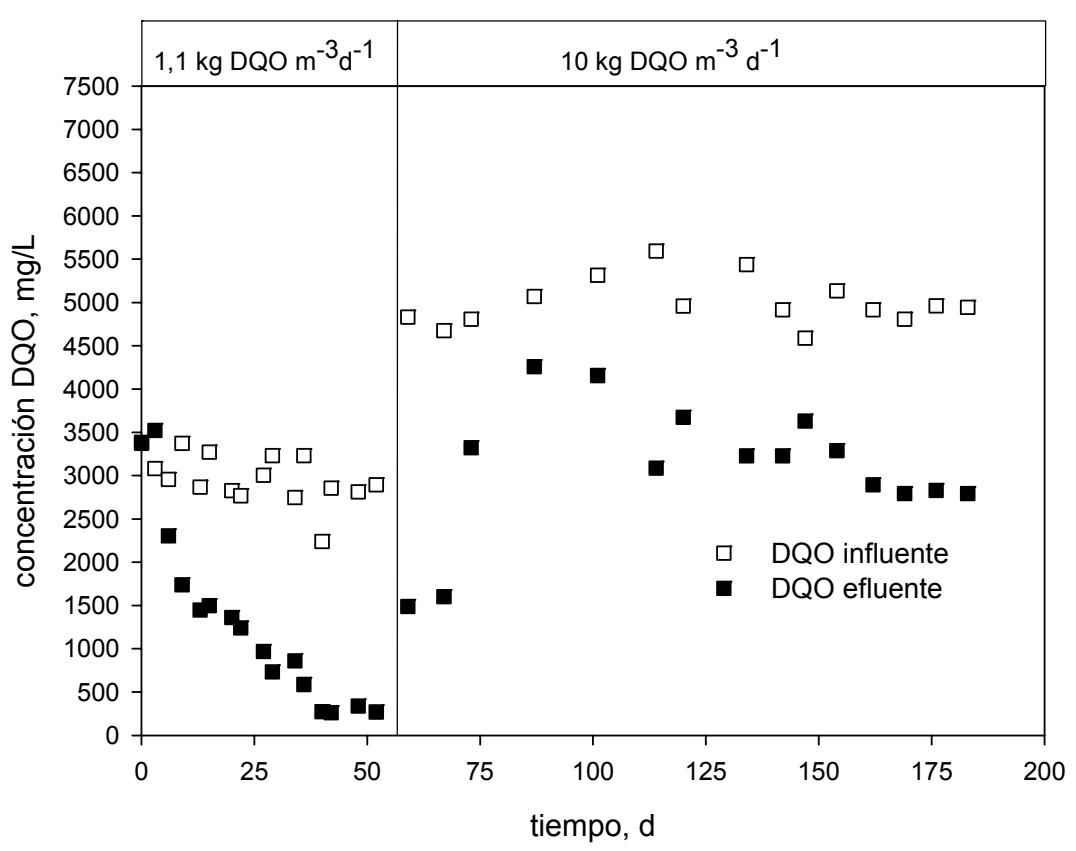

Fig. 2: Variación del DQO en el tiempo para ambas etapas de experimentación, $\mathrm{T}=37^{\circ} \mathrm{C}$, relación $\mathrm{C} / \mathrm{N}=80 \mathrm{~g} \mathrm{DQO} / \mathrm{g} \mathrm{N}^{-N_{3}}{ }_{3}^{-}$.

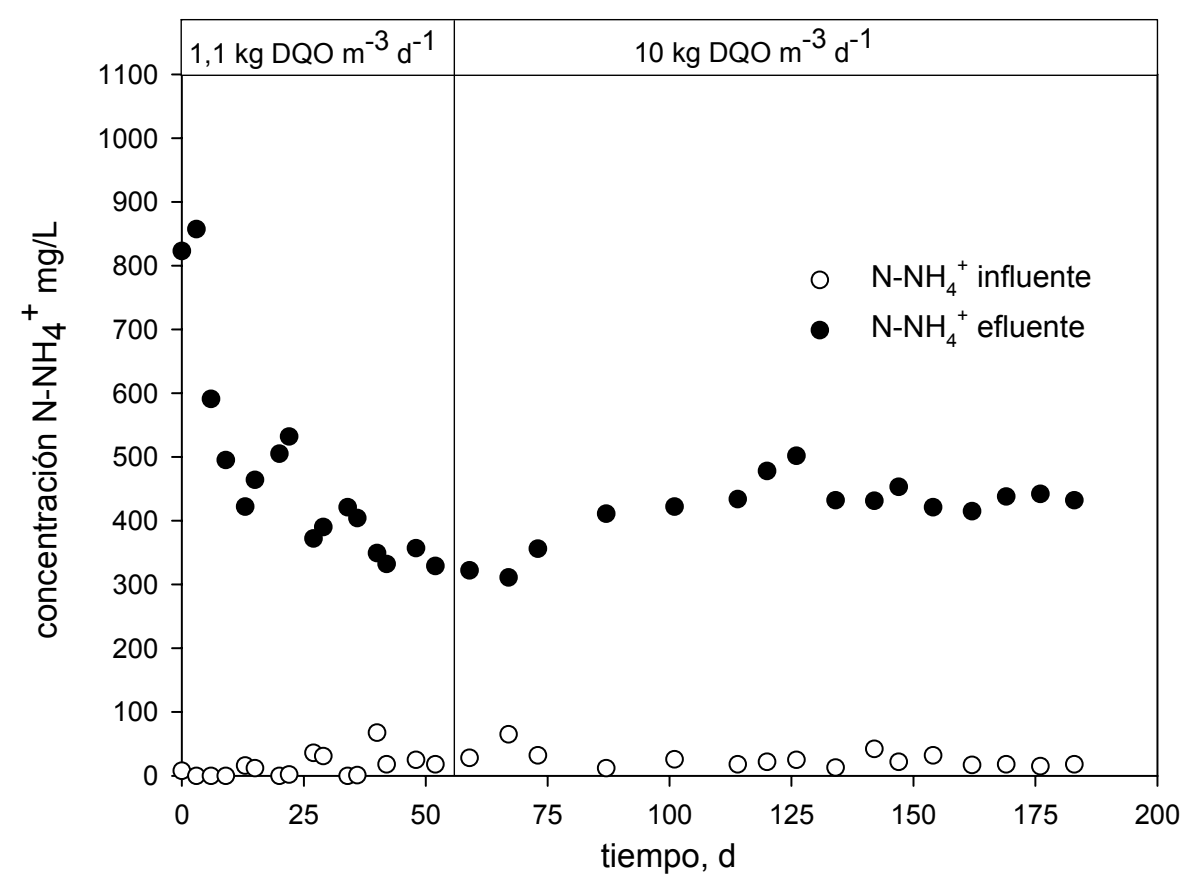

Fig. 3: Variación del $\mathrm{N}-\mathrm{NH}_{4}{ }^{+}$en el tiempo para ambas etapas de experimentación, $\mathrm{T}=37^{\circ} \mathrm{C}$, relación $\mathrm{C} / \mathrm{N}=80 \mathrm{~g} \mathrm{DQO} / \mathrm{g} \mathrm{N}^{-\mathrm{NO}_{3}}$.

En la Fig.4, se observa que la mayor parte de la remoción de carbono se produce en el primer quinto del reactor. De acuerdo a la relación $\mathrm{C} / \mathrm{N}$ estequiométrica determinada en ensayos discontinuos, 3,52 g DQO/ $\mathrm{g} \mathrm{N}^{-N_{3}}{ }_{3}^{-}$son necesarios para reducir completamente el nitrato a $\mathrm{N}_{2}$ (Soto et al., 2002), suponiendo el ácido acético como dador de electrones; por lo tanto, para reducir 36 y $64 \mathrm{mg} \mathrm{N}-\mathrm{NO}_{3}$, se necesitan 126,83 y 244 mg DQO para la desnitrificación, respectivamente. Todo el DQO restante se ocupó en la digestión anaeróbica. La menor remoción de DQO a VCO $=10 \mathrm{~kg} \mathrm{DQO} / \mathrm{m}^{3} \mathrm{~d}$ se puede atribuir a la acidificación del reactor, como lo muestra la Tabla 2. En efecto, de acuerdo a literatura (Campos, 2001), se recomienda una RA no mayor a un valor de 0,3-0,4 para evitar la 
acidificación del reactor. A VCO $=10 \mathrm{~kg} \mathrm{DQO} / \mathrm{m}^{3} \mathrm{~d}$, las RA para todas las relaciones $\mathrm{C} / \mathrm{N}$ son mayores a 0,5 , indicando un incremento de ácidos grasos volátiles (AGV) en el sistema, lo que puede inhibir el proceso de metanogénesis (Campos, 2001).

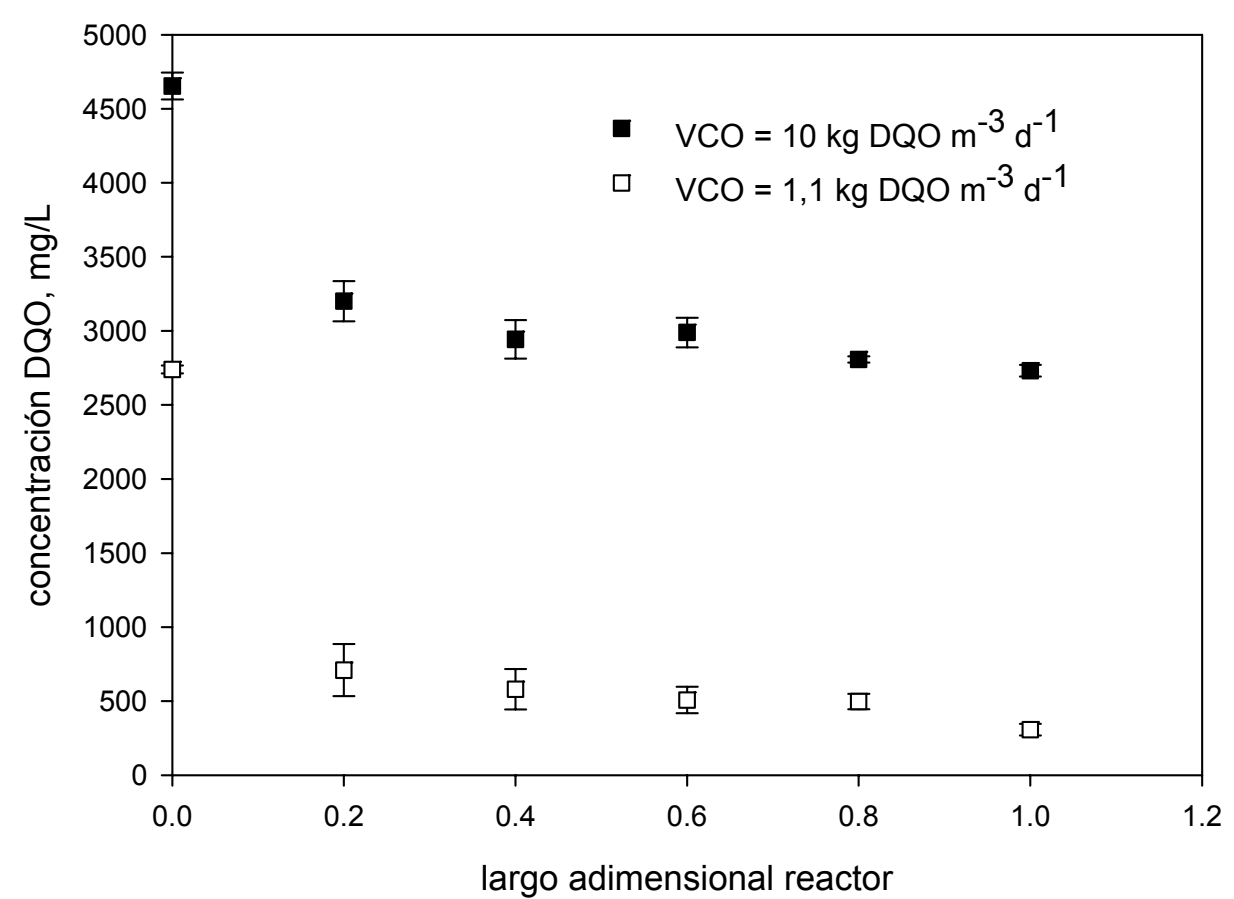

Fig 4: Perfil de DQO a lo largo del reactor tubular de lecho relleno a distintas $\mathrm{VCO}, \mathrm{T}=37^{\circ} \mathrm{C}$ y relación $\mathrm{C} / \mathrm{N}=80$.

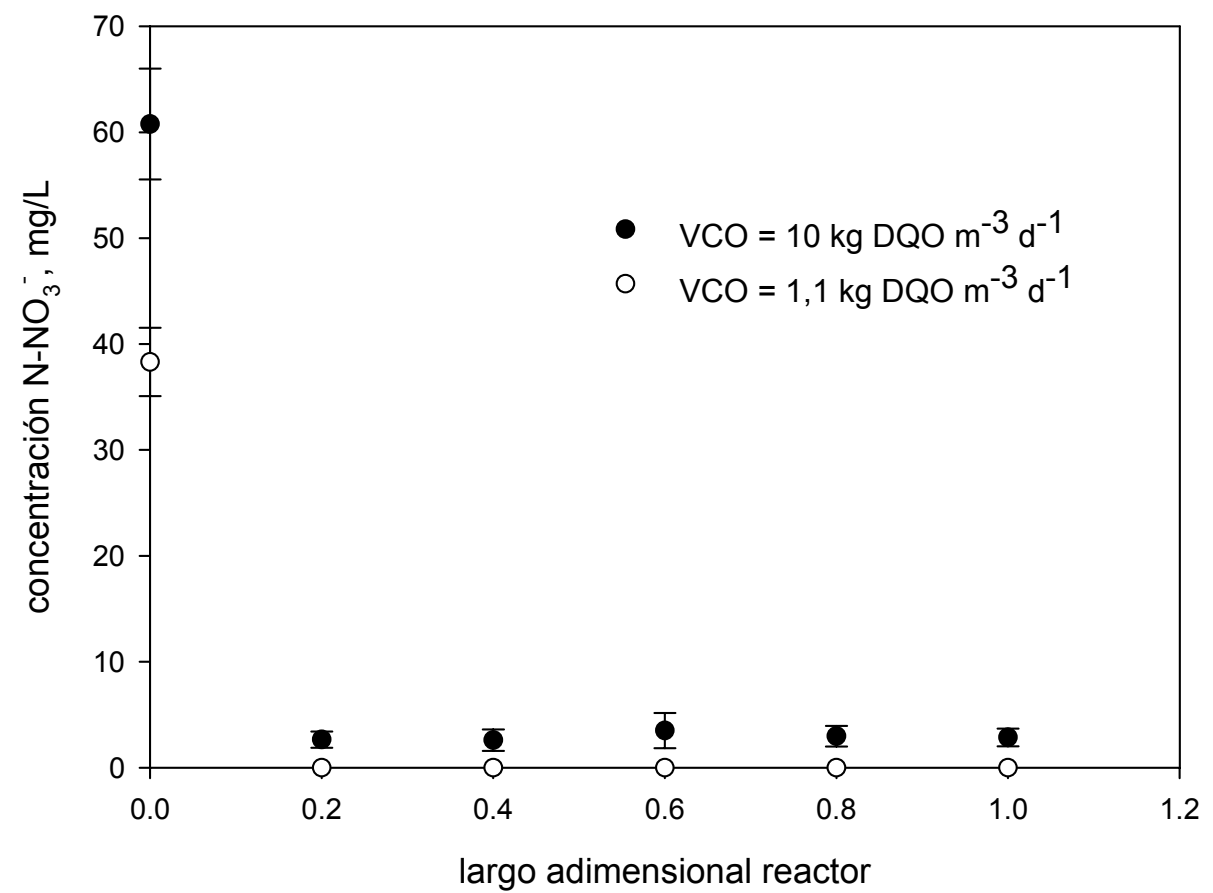

Fig 5: Perfil de $\mathrm{N}^{-N_{3}}{ }_{3}^{-}$a lo largo del reactor tubular de lecho relleno a distintas $\mathrm{VCO}, \mathrm{T}=37^{\circ} \mathrm{C}$ y relación $\mathrm{C} / \mathrm{N}=80$.

En la Fig. 5, se observa que la desnitrificación se produce en el primer quinto del reactor con una eficiencia del $100 \%$ para la etapa $1\left(\mathrm{VCO}=1,1 \mathrm{~kg} \mathrm{DQO} / \mathrm{m}^{3} \mathrm{~d}\right)$ y un $98 \%$ para la etapa $2(\mathrm{VCO}=10$ $\mathrm{kg} \mathrm{DQO} / \mathrm{m}^{3} \mathrm{~d}$ ). En esta zona de reacción también ocurre la formación de amonio, cuya concentración 
promedio para las 4 relaciones $\mathrm{C} / \mathrm{N}$ estudiadas alcanza los $350 \mathrm{mg} \mathrm{N}-\mathrm{NH}_{4}{ }^{+} / \mathrm{L}$ para la primera etapa y los $400 \mathrm{mg} \mathrm{N}-\mathrm{NH}_{4}{ }^{+} / \mathrm{L}$ para la segunda etapa.

Tabla 2: Variación de la relación de alcalinidad (RA) en función de la relación C/N a distintas VCO.

\begin{tabular}{|l|l|l|l|l|}
\hline Relación C/N (g DQO/ $\left.\mathrm{g} \mathrm{N}-\mathrm{NO}_{3}{ }^{-}\right)$ & 18 & 39 & 80 & 142 \\
\hline $\mathrm{VCO}=1,1 \mathrm{~kg} \mathrm{DQO} / \mathrm{m}^{3} \mathrm{~d}$ & 0,28 & 0,36 & 0,31 & 0,33 \\
\hline $\mathrm{VCO}=10 \mathrm{~kg} \mathrm{DQO} / \mathrm{m}^{3} \mathrm{~d}$ & 0,54 & 0,55 & 0,64 & 0,7 \\
\hline
\end{tabular}

También se observó que, para todo el rango $\mathrm{C} / \mathrm{N}$ utilizado, se produjo desnitrificación completa por sobre la RDNA, obteniéndose resultados prácticamente invariantes entre las 4 relaciones $\mathrm{C} / \mathrm{N}$ para ambas etapas. Estos resultados no coinciden con los obtenidos por otros autores en ensayos discontinuos (Akunna et al., 1994; Akunna et al., 1992), los que señalaban que a medida que se aumentaba la relación $\mathrm{C} / \mathrm{N}$ por sobre la estequiométrica, se favorecía la RDNA sobre la desnitrificación al utilizar materia orgánica fermentable. Sin embargo, en dichos estudios se utilizó un cultivo con biomasa suspendida, por lo que había una directa competencia por el nitrógeno entre las bacterias desnitrificantes y amonificantes, lo que no sucede en este estudio, donde los reactores son de bacterias adheridas. En efecto, la no existencia de RDNA en reactores de biomasa adherida también fue observada por Ruiz et al. (2006), quienes utilizando glucosa como fuente de carbono mostraron que a diferentes razones $\mathrm{C} / \mathrm{N}$, no se produjo RDNA. Por otro lado, dado que el proceso de RDNA es más rápido que el proceso de desnitrificación (Yin et al., 2002), no se puede atribuir la preferencia del proceso de desnitrificación por sobre la RDNA a factores cinéticos. Por consiguiente, se piensa que se produce efecto de la relación $\mathrm{C} / \mathrm{N}$ sobre el funcionamiento del reactor, dependiendo fundamentalmente del tipo de biomasa, es decir, biomasa suspendida o biomasa fija. Para confirmar esta hipótesis, se realizaron los balances de masa de nitrógeno total amoniacal. Las Tablas 3 y 4 muestran el balance de $\mathrm{N}-\mathrm{NH}_{4}{ }^{+}$realizado para las 2 etapas. Se observa que la principal fuente de $\mathrm{N}$ $\mathrm{NH}_{4}{ }^{+}$es el nitrógeno proveniente de la acidogénesis (nitrógeno orgánico), existiendo producción de $\mathrm{N}-\mathrm{NH}_{4}{ }^{+}$por RDNA en porcentajes menores al $4 \%$, lo que se puede suponer despreciable. La amonificación observada es similar a la obtenida por Vidal et al. (1997), quienes para la digestión anaeróbica de vertidos pesqueros con concentraciones proteicas entre 3 y 5 ( $/ \mathrm{L} / \mathrm{L})$, observaron amonio en el efluente tratado entre 0,27 y $0,46(\mathrm{~g} / \mathrm{L})$. De esta manera, se confirmó que la ruta principal del $\mathrm{N}^{-\mathrm{NO}_{3}}{ }^{-}$es la desnitrificación para las condiciones estudiadas.

La Fig. 6 muestra el perfil del pH al interior del reactor para las 2 condiciones estudiadas. El perfil para las demás relaciones $\mathrm{C} / \mathrm{N}$ es similar. Se aprecia que no hay un cambio significativo entre ambas condiciones, obteniéndose valores cercanos al neutro. El rango de variación del pH del efluente $(6,7-$ $7,5)$ indica que, bajo las condiciones estudiadas, el proceso de desnitrificación-digestión anaeróbica no necesita un control de $\mathrm{pH}$, situación ventajosa en relación al proceso en dos etapas, donde se requiere un sistema doble de regulación del $\mathrm{pH}$, uno para cada proceso, involucrando un alto consumo de ácido (para la desnitrificación) y base (para la acidogénesis).

Tabla 3: Balance de $\mathrm{N}-\mathrm{NH}_{4}{ }^{+}$en los reactores a $\mathrm{VCO}=1,1 \mathrm{~kg} \mathrm{DQO} / \mathrm{m}^{3} \mathrm{~d}$ y distintas relaciones $\mathrm{C} / \mathrm{N}$

\begin{tabular}{|c|c|c|c|c|}
\hline & $\begin{array}{c}\text { Relación } \mathrm{C} / \mathrm{N}= \\
18\end{array}$ & $\begin{array}{l}\text { Relación } \mathrm{C} / \mathrm{N}= \\
39\end{array}$ & $\begin{array}{c}\text { Relación } \mathrm{C} / \mathrm{N}= \\
80\end{array}$ & $\begin{array}{c}\text { Relación } \mathrm{C} / \mathrm{N}= \\
142\end{array}$ \\
\hline \multicolumn{5}{|c|}{ Masa Entrada $(\mathrm{mg} / \mathrm{d})$} \\
\hline Masa N-NH${ }_{4}^{+}$ & 33,75 & 35,63 & 24,38 & 33,75 \\
\hline \multicolumn{5}{|c|}{ Masa Salida (mg/d) } \\
\hline Masa N-NH${ }_{4}^{+}$salida & 615 & 682,5 & 641,25 & 661,88 \\
\hline $\begin{array}{c}\text { Masa N-NH}{ }_{4}^{+} \\
\text {por acidogénesis }\end{array}$ & 577,58 & 620,37 & 607,03 & 613,53 \\
\hline $\begin{array}{c}\text { Masa } \mathrm{N}_{-\mathrm{NH}_{4}{ }^{+}} \text {por } \\
\text { RDNA }\end{array}$ & 3,68 & 26,51 & 9,84 & 14,6 \\
\hline$\% \mathrm{~N}_{-} \mathrm{NH}_{4}^{+}$por RDNA & 0,6 & 3,9 & 1,53 & 2,21 \\
\hline
\end{tabular}


Tabla 4: Balance de $\mathrm{N}-\mathrm{NH}_{4}{ }^{+}$en los reactores a $\mathrm{VCO}=10 \mathrm{~kg} \mathrm{DQO} / \mathrm{m}^{3} \mathrm{~d}$ y distintas relaciones $\mathrm{C} / \mathrm{N}$.

\begin{tabular}{|c|c|c|c|c|}
\hline & $\begin{array}{c}\text { Relación C/N = } \\
18\end{array}$ & $\begin{array}{c}\text { Relación C/N = } \\
39\end{array}$ & $\begin{array}{c}\text { Relación C/N = } \\
80\end{array}$ & $\begin{array}{c}\text { Relación C/N = } \\
142\end{array}$ \\
\hline \multicolumn{5}{|c|}{ Masa Entrada $(\mathrm{mg} / \mathrm{d})$} \\
\hline Masa N-NH \\
\hline \multicolumn{5}{|c|}{}
\end{tabular}

Desempeño de los reactores en función de la relación C/N y la VCO

La Fig. 7 muestra el efecto de la relación $\mathrm{C} / \mathrm{N}$ sobre la remoción de materia orgánica en los reactores, indicando que la VCO tiene un mayor efecto que la relación $\mathrm{C} / \mathrm{N}$ sobre la eficiencia en la remoción de materia orgánica, observándose diferencias del orden del $10 \%$ a diferentes relaciones $\mathrm{C} / \mathrm{N}$. La VCO incidió principalmente en la etapa de digestión anaeróbica, dado que la desnitrificación no se ve afectada por el aumento de la concentración de materia orgánica.

La Fig. 7 permite observar que la remoción de materia orgánica a VCO $=1,1 \mathrm{~kg} \mathrm{DQO} / \mathrm{m}^{3} \mathrm{~d}$ alcanza valores entre el 90 y el $84,4 \%$, cercanos a valores obtenidos por Garrido et al. (2001) y MosqueraCorral et al. (2001), como se muestra también en la Tabla 5. Sin embargo, estos estudios trabajaron a bajas relaciones $\mathrm{C} / \mathrm{N}$, lo que incide en la mayor cantidad de materia orgánica reducida por desnitrificación. Cuando se aumentó la VCO, el reactor bajó su eficiencia al $50 \%$, sin embargo, este comportamiento es mejor que el presentado por Kim et al. (2004), que logró un pobre desempeño en la remoción de materia orgánica a pesar de trabajar a bajas relaciones $\mathrm{C} / \mathrm{N}$. De esta forma, se puede decir que el sistema trabajado es uno de los más eficientes en la remoción de materia orgánica por digestión anaeróbica, aún cuando se trabajó a altas relaciones $\mathrm{C} / \mathrm{N}$.

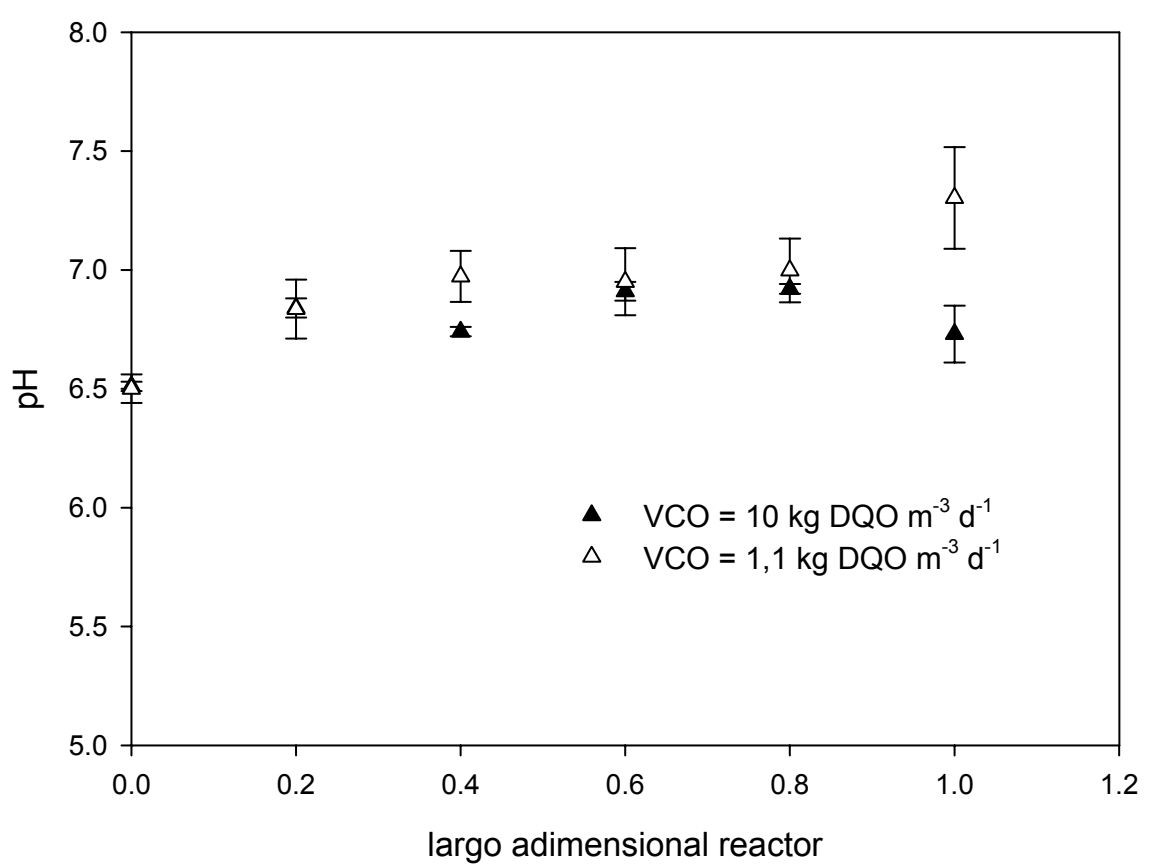

Fig 6: Perfil de $\mathrm{pH}$ a lo largo del reactor tubular de lecho relleno a distintas $\mathrm{VCO}, \mathrm{T}=37^{\circ} \mathrm{C}$ y relación $\mathrm{C} / \mathrm{N}=80$. 


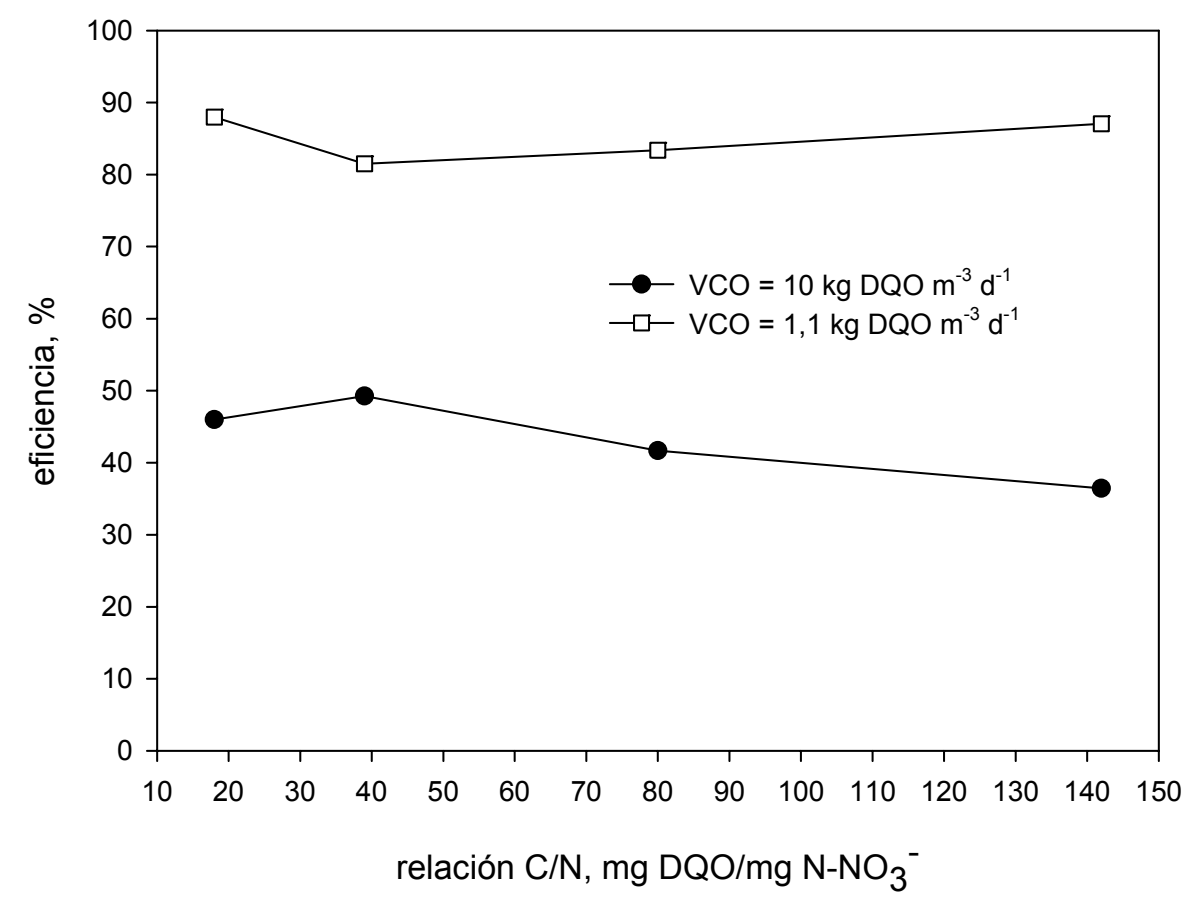

Fig 7: Eficiencia en la remoción de materia orgánica a distintas relaciones $\mathrm{C} / \mathrm{N}$ en reactores tubulares de lecho relleno a $37^{\circ} \mathrm{C}$ alimentados con concentraciones entre 2800 y $5000 \mathrm{mg} \mathrm{DQO} / \mathrm{L}$.

Otro parámetro que puede afectar la eficiencia de remoción en el proceso es la temperatura (Choorit y Wisarnwan, 2007). Para residuos salinos, Aspé et al. (1997) determinaron, a través del cálculo de energías de activación, que la temperatura en el tratamiento residuos industriales salinos es menos importante que en el tratamiento de residuos normales. En efecto, el incremento de la temperatura desde 20 a $30^{\circ} \mathrm{C}$ incide en un aumento de 2,43 veces en la producción de metano si se utiliza un lodo digestor adaptado, mientras que para un sedimento marino, como el utilizado en este trabajo, el incremento es sólo de 1,46 veces (Aspé et al., 1997). Choorit y Wisarnwan, (2007) estudiaron el efecto de la temperatura y la VCO sobre la digestión anaeróbica de efluentes de la producción de aceite de palma, mostrando que un cambio en la VCO tiene un efecto mayor sobre la producción de metano y sobre la reducción de DQO. Del análisis de sus resultados se pudo determinar que el incremento desde 37 a $43^{\circ} \mathrm{C}$ produce una variación del 8,5\% en la reducción de DQO y un $11,5 \%$ en la producción de metano, mientras que el incremento desde 9,72 a 12,25 g DQO/L d produce una variación del $48 \%$ en la reducción de DQO y un $28 \%$ en la producción de metano. Para confirmar estos resultados, se realizó un análisis de sensibilidad de los parámetros temperatura (T) y VCO sobre la eficiencia de reducción de materia orgánica. De la literatura, se obtuvieron las siguientes relaciones:

Eficiencia de reducción de materia orgánica en función de la Temperatura (Young y Yang, 1989):

$E_{T}=E_{(30)} \cdot t^{(T-30)}$

donde $E_{T}$ es la eficiencia a una temperatura dada, $E_{(30)}$ es la eficiencia a $30^{\circ} \mathrm{C}$ y $t$ es un coeficiente de temperatura, que toma el valor de 1,01-1,02. El rango de T válido está entre $20-40^{\circ} \mathrm{C}$.

Eficiencia de reducción de materia orgánica en función de la VCO (Sánchez et al., 2005b):

$E_{V C O}=e^{-k \cdot V C O} \cdot 100$

donde $E_{V C O}$ es la eficiencia a una VCO dada y $k$ es una constante de ajuste $(0,21)$. El rango de VCO válido para la ecuación (10) está entre 0 y $12 \mathrm{~g} \mathrm{DQO/L} \mathrm{d.}$ 
Con las ecuaciones 9 y 10, se realizó el siguiente análisis, basado en el trabajo de Wu et al. (2005). Variando la $T\left(\triangle T / T_{0}\right)$ y VCO $\left(\triangle V C O / V C O_{0}\right)$ desde un valor base, se calculó la variación de $E$ con respecto a un valor base, $E_{0}\left(\Delta E / E_{0}\right)$. Para estos casos, se utilizaron los siguientes valores: $T_{0}=$ $20^{\circ} \mathrm{C}, E_{T, 0}=73 \%, V_{C O}=4,5 \mathrm{~kg} \mathrm{DQO} / \mathrm{m}^{3} \mathrm{~d}, E_{V C O, 0}=39 \%$. El rango de variación de $\mathrm{T}$ y $V C O$ fue \pm $40 \%$. Los resultados se muestran en la Fig. 8.

En la Fig. 8 se aprecia que la temperatura tiene un menor efecto que la VCO sobre la eficiencia de remoción de DQO. Por ejemplo, para una variación de $-20 \%$ de $\mathrm{T}$, la eficiencia cae un $10 \%$, mientras que el mismo porcentaje de variación de VCO produce un cambio en la eficiencia del $23 \%$. De esta forma, se puede concluir que el efecto de la VCO es mayor que el efecto de la temperatura en el proceso estudiado.

Las velocidades de remoción de nitrato obtenidas fluctuaron entre 0,008 y 0,58 $\mathrm{g} \mathrm{N}-\mathrm{NO}_{3}{ }^{-} / \mathrm{L}$ d para concentraciones de 20 y $280{\mathrm{mg} \mathrm{N}-\mathrm{NO}_{3}}^{-} / \mathrm{L}$, respectivamente. Se observó que la velocidad de remoción de nitrato disminuye a medida que aumenta la relación $\mathrm{C} / \mathrm{N}$, debido a que la cantidad de nitrato alimentado disminuye. La velocidad máxima de remoción de $\mathrm{N}-\mathrm{NO}_{3}{ }^{-}$está dentro de los valores reportados en la literatura (Kim et al., 2004; Garrido et al., 2001; Fang y Zhou, 1999), siendo muy parecida a la reportada por Fang y Zhou (1999) y menor que los valores reportados por Kim et al. (2004) e Im et al. (2001). Las menores velocidades de remoción obtenidas en este estudio se pueden atribuir a la utilización de materia orgánica de mayor peso molecular y menor biodegradabilidad. Sin embargo, si se aumenta la concentración de $\mathrm{N}_{-} \mathrm{NO}_{3}{ }^{-}$a valores similares a las concentraciones de $\mathrm{N}$ $\mathrm{NH}_{4}{ }^{+}$de salida, la VRN máxima se puede incrementar. En efecto, datos aún no publicados mostraron

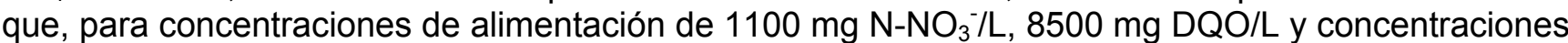
de $\mathrm{N}-\mathrm{NH}_{4}{ }^{+}$de salida de $1200 \mathrm{mg} \mathrm{N}-\mathrm{NH}_{4}{ }^{+} / \mathrm{L}$, se obtiene una remoción total de nitratos, con una velocidad de $1,16 \mathrm{~kg} \mathrm{~N}-\mathrm{NO}_{3}{ }^{-} / \mathrm{m}^{3} \mathrm{~d}$.

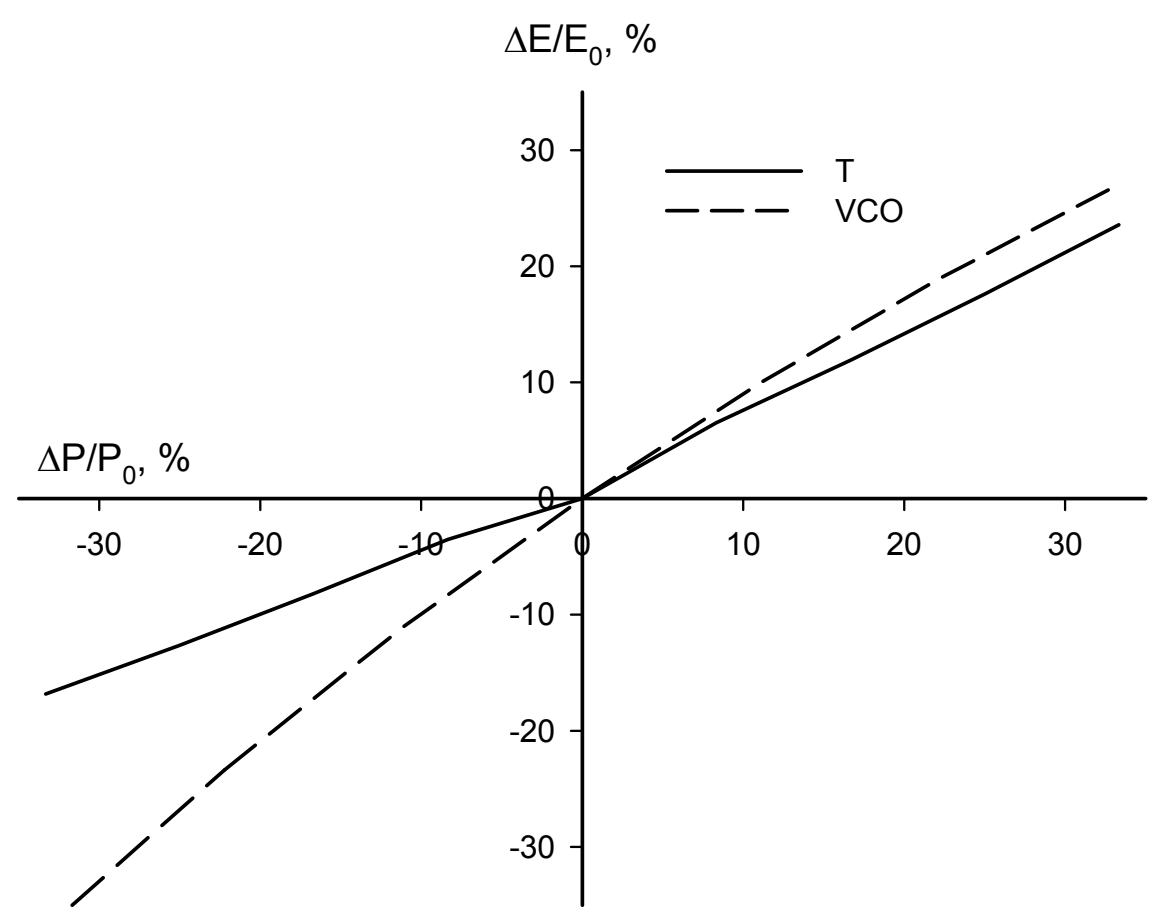

Fig. 8: Análisis de sensibilidad de parámetros, $\Delta P / P_{0} . P$ representa la T ó VCO.

Por otro lado, en este trabajo se obtuvieron velocidades de remoción mayores que las encontradas por Mosquera-Corral et al. (2001), quienes utilizando vertidos de las conservas de pescado, para una VCN entre $1-3 \mathrm{~kg} \mathrm{~N}-\mathrm{NO}_{3}-1 \mathrm{~m}^{3} \mathrm{~d}$, obtuvo una velocidad de remoción de $0,22\left(\mathrm{~kg} \mathrm{~N}^{-\mathrm{NO}_{3}-} / \mathrm{m}^{3}\right.$ día), valor que es menor en 2,6 veces con respecto al encontrado en este trabajo. De esta manera, el sistema propuesto logra eficiencias altas en cuanto a la remoción de $\mathrm{N}^{-\mathrm{NO}_{3}}{ }_{3}^{-}$. 
Tabla 5: Resultados obtenidos en éste y otros trabajos para la desnitrificación-digestión anaeróbica. *(g COT/ $\left.\mathrm{g} \mathrm{N}^{-\mathrm{NO}_{3}}{ }^{-}\right)$.

\begin{tabular}{|c|c|c|c|c|c|c|c|}
\hline Referencia & $\begin{array}{c}\mathrm{VCN} \\
\left(\mathrm{kg} \mathrm{N}^{-}\right. \\
\mathrm{NO}_{3}^{-} / \mathrm{m}^{3} \\
\text { d) }\end{array}$ & $\begin{array}{c}\text { VRN } \\
\text { máxima } \\
(\mathrm{kg} \mathrm{N}- \\
\mathrm{NO}_{3}^{-} / \mathrm{m}^{3} \\
\text { d) }\end{array}$ & $\begin{array}{c}\mathrm{VCO} \\
(\mathrm{kg} \\
\mathrm{DQO} / \mathrm{m}^{3} \\
\mathrm{~d})\end{array}$ & $\begin{array}{c}\text { Relación } \\
\mathrm{C} / \mathrm{N} \\
(\mathrm{g} \mathrm{DQO} / \mathrm{g} \\
\left.\mathrm{N}^{-\mathrm{NO}_{3}}{ }^{-}\right)\end{array}$ & $\begin{array}{l}\text { TRH } \\
\text { (d) }\end{array}$ & $\begin{array}{l}\text { Eficiencia } \\
\mathrm{NO}_{x}(\%)\end{array}$ & $\begin{array}{l}\text { Eficiencia } \\
\text { COT (\%) }\end{array}$ \\
\hline $\begin{array}{c}\text { Mosquera-Corral } \\
\text { et al. (2001) }\end{array}$ & $\begin{array}{l}0,11- \\
0,213\end{array}$ & 0,22 & $\begin{array}{l}1,33- \\
1,67\end{array}$ & $2-3^{*}$ & 0,75 & $53-95$ & 80 \\
\hline Kim et al. (2004) & $2-4$ & 2,85 & $\begin{array}{l}4,35- \\
8,69\end{array}$ & 2 & 0,23 & $40-60$ & $45-65$ \\
\hline $\begin{array}{c}\text { Fang y Zhou } \\
\text { (1999) }\end{array}$ & $0,25-6$ & 0,6 & $2-0,83$ & $2-5$ & 1 & 100 & $80-90$ \\
\hline $\begin{array}{l}\text { Garrido et al. } \\
(2001)\end{array}$ & 0,75 & 0,75 & $5,5-3,3$ & $3,5-4,2$ & $0,5-1$ & $90-78$ & 94 \\
\hline Im et al. (2001) & 0,3 & 1,04 & $2,6-19,2$ & $10-18$ & $1,3-4$ & 99 & $80-90$ \\
\hline Este trabajo & $\begin{array}{c}0,008- \\
0,58\end{array}$ & 0,58 & $1-10$ & $18-140$ & $\begin{array}{l}0,5- \\
2,6\end{array}$ & 100 & $50-88$ \\
\hline
\end{tabular}

\section{CONCLUSIONES}

Es factible remover simultáneamente carbono y nitrato desde vertidos proteicos salinos, de alta carga orgánica y sin pretratamiento, utilizando reactores tubulares de biomasa adherida. El comportamiento de cuatro reactores filtro estudiados es similar, no observándose efecto de la relación $\mathrm{C} / \mathrm{N}$ sobre los perfiles de concentración y sobre la eficiencia del proceso, realizándose la mayor parte del proceso en el primer quinto de los reactores. El amonio se produce mayormente por la digestión anaeróbica de la materia orgánica, no existiendo RDNA para las condiciones estudiadas. El sistema logró eficiencias superiores al $98 \%$ en cuanto a la remoción de $\mathrm{N}-\mathrm{NO}_{3}{ }^{-}$y N-NO${ }_{2}^{-}$para todas las condiciones estudiadas y superiores al $80 \%$ en cuanto a la remoción de materia orgánica para VCO $=1,1 \mathrm{~kg}$ $\mathrm{DQO} / \mathrm{m}^{3}$ d. El parámetro que más afecta el proceso de remoción de materia orgánica vía desnitrificación/digestión anaeróbica es la velocidad de carga orgánica (VCO). A menor VCO, mayor eficiencia en la remoción de materia orgánica.

\section{AGRADECIMIENTOS}

Este trabajo fue financiado por Proyecto 1040495, FONDECYT (Chile) y por la Beca AT-23070102.

\section{REFERENCIAS}

Akunna, J.C. y otros cuatro autores; Combined Organic-Carbon and Complete Nitrogen Removal Using Anaerobic and Aerobic Upflow Filters, Water Science and Technology: 30(12), 297-306 (1994).

Akunna, J.C., C. Bizeau y R. Moletta; Denitrification in Anaerobic Digesters - Possibilities and Influence of Waste-Water Cod/N-Nox Ratio, Environmental Technology: 13(9), 825-836 (1992).

Akunna, J.C., C. Bizeau y R. Moletta; Nitrate Reduction by Anaerobic Sludge Using Glucose at Various Nitrate Concentrations - Ammonification, Denitrification and Methanogenic Activities, Environmental Technology: 15(1), 41-49 (1994).

APHA, AWWA, WPCF; Standard Methods for the Examination of Water and Wastewater, 18th edition, Washington, USA (1992). 
Aspe, E., M.C. Marti y M. Roeckel; Anaerobic treatment of fishery wastewater using a marine sediment inoculum, Water Research: 31(9), 2147-2160 (1997).

Baloch, M.I., J.C. Akunna y P.J. Collier; Carbon and nitrogen removal in a granular bed baffled reactor, Environmental Technology: 27(2), 201-208 (2006).

Bernet, N. y otros cuatro autores; Combined anaerobic-aerobic sbr for the treatment of piggery wastewater, Water Research: 34(2), 611-619 (2000).

Bernet, N., N. Delgenes y R. Moletta; Denitrification by anaerobic sludge in piggery wastewater, Environmental Technology: 17(3), 293-300 (1996a).

Bernet, N., F. Habouzit y R. Moletta; Use of an industrial effluent as a carbon source for denitrification of a high-strength wastewater, Applied Microbiology and Biotechnology: 46(1), 92-97 (1996b).

Campos, E.; Optimización de la digestión anaerobia de purines de cerdo mediante codigestión con residuos orgánicos de la industria agroalimentaria, Tesis de Doctorado, Departamento de Medio Ambiente y Ciencias del Suelo, Laboratorio de Ingeniería Ambiental, Universidad de LLeida, LleidaEspaña (2001). http://www.tesisenxarxa.net/TDX-0909105-125302/

Chen, K.C., Y.F. Lin y J.Y. Houng; Performance of a continuous stirred tank reactor with immobilized denitrifiers and methanogens, Water Environment Research: 69(2), 233-239 (1997).

Choorit W. y P. Wisarnwan; Effect of temperature on the anaerobic digestion of palm oil mill effluent, Electronic Journal of Biotechnology, ISSN: 0717-3458 (en linea), 10(3), (2007). http://www.ejbiotechnology.info/content/vol10/issue3/full/7/index.html.

Fang, H.H.P. y G.M. Zhou; Interactions of methanogens and denitrifiers in degradation of phenols, Journal of Environmental Engineering-Asce: 125(1), 57-63 (1999).

Garrido, J. M., R. Mendez y J.M. Lema; Simultaneous urea hydrolysis, formaldehyde removal and denitrification in a multifed upflow filter under anoxic and anaerobic conditions, Water Research: 35(3), 691-698 (2001).

Gonzalez, G., H Urrutia, M. Roeckel y E. Aspe; Protein hydrolysis under anaerobic, saline conditions in presence of acetic acid, Journal of Chemical Technology and Biotechnology: 80(2), 151-157 (2005).

Hendriksen, H. V. y B.K. Ahring; Integrated removal of nitrate and carbon in an upflow anaerobic sludge blanket (uasb) reactor: Operating performance, Water Research: 30(6), 1451-1458 (1996).

Im, J. H. y otros cuatro autores; Simultaneous organic and nitrogen removal from municipal landfill leachate using an anaerobic-aerobic system, Water Research: 35(10), 2403-2410 (2001).

Kim, S., H. Jung, K.S. Kim y I.S. Kim; Treatment of high nitrate-containing wastewaters by sequential heterotrophic and autotrophic denitrification, Journal of Environmental Engineering-Asce: 130(12), 1475-1480 (2004).

Mosquera-Corral, A. y otros cuatro autores; Simultaneous methanogenesis and denitrification of pretreated effluents from a fish canning industry, Water Research: 35(2), 411-418 (2001).

Ripley L.E., W.C. Boyle y J.C. Converse; Improved alkalimetric monitoring for anaerobic digestion of high-strength wastes, Journal of Water Pollution Control Federation: 58(5), 406-411 (1986).

Ruiz, G., D. Jeison y R. Chamy; Development of denitrifying and methanogenic activities in USB reactors for the treatment of wastewater: Effect of COD/N ratio, Process Biochemistry: 41(6), 13381342 (2006). 
Sánchez E. y otros cuatro autores; Effect of organic loading rate on the stability, operational parameters and performance of a secondary upflow anaerobic sludge bed reactor treating piggery waste, Bioresource Technology: 96, 335-344 (2005a).

Sánchez, O., E. Aspe, M.C. Marti y M. Roeckel; Rate of ammonia oxidation in a synthetic saline wastewater by a nitrifying mixed-culture, Journal of Chemical Technology and Biotechnology: 80(11), 1261-1267 (2005b).

Soto, O., O. Sánchez, E. Aspe y M. Roeckel; Denitrification kinetics of simulated fish processing wastewater at different ratios of nitrate to biomass, Biotechnology Letters: 24(14), 1173-1176 (2002).

Vidal, G., E. Aspe, M.C. Marti y M. Roeckel; Treatment of recycled wastewaters from fishmeal factory by an anaerobic filter, Biotechnology Letters: 19(2), 117-121 (1997).

Wu C., J. Huang y $\mathrm{H}$. Gou; Expansion characteristics of an anaerobic fluidized bed reactor with internal biogas production, Journal of Chemical Technology and Biotechnology: 80(11), 1273-1281 (2005).

Yin S.X., D. Chen, L.M. Chen y R. Edis; Dissimilatory nitrate reduction to ammonium and responsible microorganisms in two Chinese and Australian paddy soils, Soil Biology \& Biochemistry 34, 11311137 (2002).

Young J.C. y B.S. Yang; Design considerations for full scale anaerobic filters, Journal of Water Pollution and Control Federation: 61(9), 1576-1587 (1989). 